\title{
CALIBRATION OF POSSUM DENSITY ESTIMATES FROM RAISED LEG-HOLD TRAPS
}

\author{
M.D. THOMAS ${ }^{1}$ and J.A. BROWN ${ }^{2}$
}

${ }^{1}$ Pest Control Research, P.O. Box 7223, Christchurch, New Zealand

${ }^{2}$ University of Canterbury, Private Bag 4800, Christchurch, New Zealand

Corresponding author: malcolm.thomas@pcr.co.nz

\begin{abstract}
We undertook field trials to compare differences between ground- and raised-set leg-hold traps to measure population abundance of the brushtail possum (Trichosurus vulpecula). We discuss the observed differences in trap catch rates and the use, and potential misuse, of a calibration index to 'correct' the raised-set estimates so they can be compared with the ground-set estimates.
\end{abstract}

Keywords: possums, Trichosurus vulpecula, monitoring, trapping, vertebrate pest control.

\section{INTRODUCTION}

Effective possum (Trichosurus vulpecula) control in New Zealand needs standard methods to estimate possum density. These estimates can be compared regionally to identify priority areas requiring possum control. A standard method has been developed for possum monitoring (NPCA 2001), using the number of possums captured on lines of leg-hold traps set on the ground (ground-sets). The estimates are usually made after possum control has been undertaken and are referred to as the residual trap-catch (RTC). Residual refers to the remaining population and is commonly targeted at $5 \%$, but can be as low as $0.5 \%$. These low residual population levels are targeted with the aim of significantly reducing damage to the native flora and fauna or the incidence of bovine tuberculosis in cattle and deer herds.

Unfortunately the use of leg-hold traps puts flightless birds, such as the native weka (Gallirallus australis) and kiwi (Apteryx spp.), at risk of being maimed or killed. Consequently, when the Department of Conservation (DOC) undertakes possum monitoring in areas where these birds are present, traps are often placed above ground (raised-sets). Guidelines used by DOC for raised-sets are based on results from a trapping programme to eradicate possums from Kapiti Island (Sherley 1992) and on bait station trials carried out on kiwi in the Wellington Zoo (A.D. Robinson, unpubl. data). The guidelines specify that traps are to be raised to a height of $700 \mathrm{~mm}$ although results from subsequent trials suggest that $700 \mathrm{~mm}$ may not be high enough to prevent the capture of weka (Thomson et al. 1996).

It is generally perceived that raised-sets do not capture as many possums as groundsets. The concept of a calibration index to correct the lower catch rates in raised-sets, so the RTC estimates are directly comparable to ground-sets, has been discussed within the possum industry. Indices are often given a high status by biologists in their belief that the index is a measure of the true population size, or true population change. An important, but perhaps subtle, point is lost in this thinking - an index is only an estimate, it is not the truth (Brown \& Hickling 2000). If an index were to be used for calibrating raised-set RTC to ground-sets, such an index would be an estimate of the true calibration for the specific possum monitoring operation. The index, therefore, should have a measure of uncertainty about it. This uncertainty is measured (more correctly it is estimated) in the indices' variance. The variance (and confidence interval) of the calibrated RTC should include firstly, a measure of the uncertainty of the raised-set RTC and secondly, the measure of uncertainty of the calibration index.

New Zealand Plant Protection 54:71-75 (2001) 
This study was undertaken to determine whether there are differences in catches between ground- and raised-sets. The feasibility of producing a calibration index to correct for any differences in catch rates was also investigated.

\section{MATERIALS AND METHODS}

Field trials were undertaken at three study sites (approximately 500 ha) in habitats that represented areas where possum monitoring is undertaken. These were: beech forest in the Eglinton and Hollyford Valleys, Fiordland, (11-17 December 1999), mixed podocarp forest at Rotoehu Forest, Rotorua (21-23 January 2000) and forest/pasture margin at Galatea (6-9 March 2000). The sites were chosen because they had recently undergone possum control and were known to contain low possum numbers characteristic of many possum monitoring operations.

The standard possum monitoring protocol (NPCA 2001) was used to establish ten trap-lines in each study site. Lines were set out by following a compass bearing and were at least $200 \mathrm{~m}$ apart. A spacing of $200 \mathrm{~m}$ is given in the protocol to ensure that possum captures on one line have minimal influence on catch rates on adjacent lines (NPCA 2001). We used lines of 21 Victor No. 1 traps, (M. Woodcraft, 128 Marine Parade, Mt Maunganui) and we tested three different set heights within each line, i.e. 7 traps for each set height. The protocol specifies 10 lines of 10 traps for the size of the study sites we used (approximately 500 ha). We used a design with 10 lines of 7 traps for each set height, which is slightly less than that specified in the protocol. Traps were all $20 \mathrm{~m}$ apart, as measured by a hip chain. Trap heights were either the standard ground-set, as specified in the protocol (NPCA 2001), or raised-sets at $350 \mathrm{~mm}$ or $700 \mathrm{~mm}$ heights. The height of $350 \mathrm{~mm}$ was used because DOC staff in some areas use this height in preference to the $700 \mathrm{~mm}$ height (Thomas \& Brown 2000). Trap-heights within groups of three were randomly allocated using a list of random numbers to eliminate possible biases that could occur from setting certain trap-sets at favoured sites. The raised-sets at the beech and mixed podocarp sites were located on trees, and the raised-sets at the forest/pasture site were located on fence posts.

Metal L-brackets were used to raise the traps. These brackets had two prongs that fitted into the coil springs on the trap to hold it firmly. The prongs allowed the L-bracket to be bent so that the trap could remain horizontal regardless of the angle of the tree. All raised-sets had $400 \mathrm{~mm}$ chains that were nailed halfway between the ground and trap so that captured possums could fall to the ground after capture. Flour lure was used to attract possums to the traps. Flour placement was as specified in the protocol (NPCA 2001 ) for the ground-sets. For the raised-sets flour was placed $100 \mathrm{~mm}$ from the ground up to and beside the trap. A further 'white blaze' was placed $100 \mathrm{~mm}$ above the trap.

Traps were set for three fine nights giving 630 trap-nights for each set type. Traps were checked daily and notes kept on the number of possums captured, the number of possum escapes, the number of sprung traps, and the number of non-targets captured. Estimates of RTC were calculated for each habitat type using the method described in the protocol (NPCA 2001). RTC estimates were compared to determine whether significant differences in catch rates occurred within each habitat type using analysis of variance (ANOVA).

A calibration index was calculated as the ratio of the average ground-set RTC to the average raised-set RTC:

$$
I=\frac{R T C_{\text {ground }}}{R T C_{\text {raised }}}
$$

The index was calculated for the $350 \mathrm{~mm}$ sets and for the $700 \mathrm{~mm}$ sets. Given the differences in the study sites, data from the Fiordland and Rotoehu trials were used to calculate "forest" indices and data from the Galatea trial to calculate "forest/pasture margin" indices. 


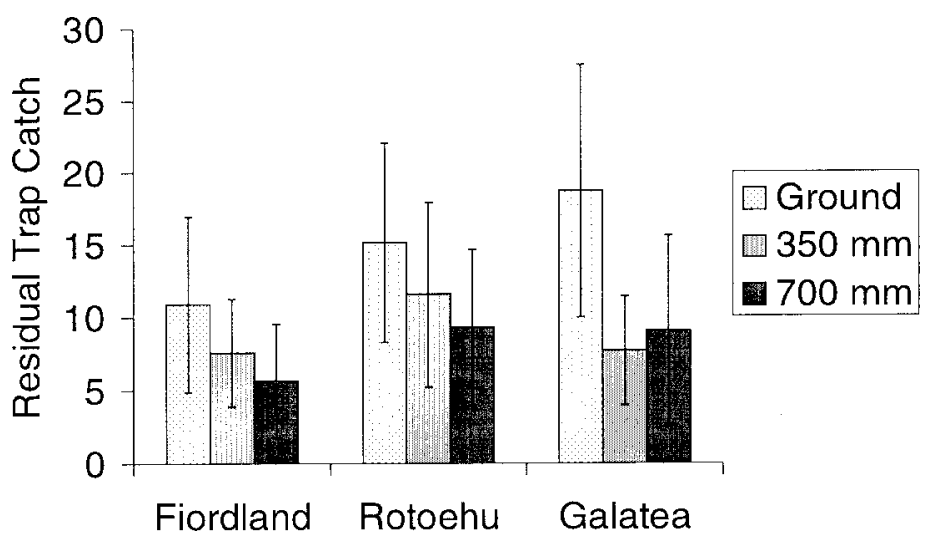

FIGURE 1: Comparison of residual trap-catches between the three trap-set heights (ground, $350 \mathrm{~mm}$ and $700 \mathrm{~mm}$ ) with $95 \%$ confidence intervals $(\mathrm{n}=10$ for each site).

\section{RESULTS}

More possums were caught in ground-set traps than in raised-set traps (Fig. 1). At Fiordland and Rotoehu more possums were caught in the $350 \mathrm{~mm}$ raised-sets than $700 \mathrm{~mm}$ raised-sets but at Galatea slightly more possums were caught in the $700 \mathrm{~mm}$ raised-sets than $350 \mathrm{~mm}$ raised-sets. The differences in catch rates between set heights were not statistically significant $(\mathrm{P}>0.05)$ at Fiordland and Rotoehu, but there were significantly more possums caught in ground-sets than $350 \mathrm{~mm}$ raised-sets at the forest/pasture margin site at Galatea $(\mathrm{P}<0.05$, Fig. 1).

The calibration indices for the raised-sets varied from 1.36 for forest sites to 2.42 for forest/pasture margin (Table 1). The variances for the indices increased with the size of the index and were larger for the forest/pasture margin where sample sizes were smaller (data was from one site whereas the data for the forest indices were from two sites).

TABLE 1: Calibration indices and variance for raised-sets.

\begin{tabular}{|c|c|c|c|c|}
\hline \multirow[b]{2}{*}{ Set height } & \multicolumn{2}{|c|}{ Forest } & \multicolumn{2}{|c|}{ Forest/pasture margin } \\
\hline & $\begin{array}{l}\text { Ratio to } \\
\text { ground }\end{array}$ & Variance & $\begin{array}{l}\text { Ratio to } \\
\text { ground }\end{array}$ & Variance \\
\hline $350 \mathrm{~mm}$ & 1.36 & 0.36 & 2.42 & 3.81 \\
\hline $700 \mathrm{~mm}$ & 1.74 & 0.78 & 2.06 & 3.07 \\
\hline
\end{tabular}

\section{DISCUSSION}

This study showed that more possums were caught in ground-set traps than in raised-set traps but the differences at the forest sites were not significantly different. Results at the forest/pasture margin site did show a significant difference between the ground-sets and the $350 \mathrm{~mm}$ raised-sets. A reason for site differences could be because the raised-sets at Galatea were located on fence posts while the Fiordland and Rotoehu raised-sets were located on trees. It is possible that there are behavioural differences associated with possums climbing fence posts compared with climbing trees. There is often confused thinking on the comparison between catch rates from ground- and raised-sets because the issue of statistical significance 
is often not fully understood. The appropriate question to be asked is not whether there is a difference in trap catch rates between ground- and raised-sets because the answer is probably yes, but whether, given the amount of survey effort that is used in monitoring, whether these differences are meaningful. This study used a level of survey effort slightly less than specified in the national protocol (10 lines of 7 traps for each trap-height compared with 10 lines of 10 traps) and found that the differences in trap catch rates were not statistically significant for the forest sites. Even with lines of ten traps we would not expect the differences to be statistically significant.

The question of whether a calibration index should be used has also been confused by not referring to such an index as being an estimate of the calibration. The calibration index is estimated and therefore the confidence interval of the new calibrated RTC needs to be widened to include the uncertainty about the estimate. As an example, consider the data from $700 \mathrm{~mm}$ set traps in Fiordland being calibrated to a ground-set catch. Ignoring that the estimated calibration index is derived from this very data set, the calibrated RTC is calculated as:

$$
\begin{aligned}
\text { calibrated RTC } & =\text { raised-set RTC } \times \text { index } \\
& =5.71 \% \times 1.74 \\
& =9.935 \%
\end{aligned}
$$

The calibrated RTC is larger than the original raised-set RTC. The next step is to estimate the confidence interval for the calibrated RTC. The half width of the $95 \%$ confidence interval for the raised-set RTC is:

$$
\begin{aligned}
\mathrm{CI}_{\text {half-width }} & =t_{0.05 / 2,9} \times \mathrm{se} \\
& =2.26 \sqrt{(29.23 / 10)} \\
& =3.86
\end{aligned}
$$

The limits of the 'un-calibrated' $95 \%$ confidence interval range from $1.85 \%$ to $9.58 \%$ $(5.71 \% \pm 3.86 \%)$. To calculate the $95 \%$ confidence interval of the calibrated RTC estimate the variance of the product of the RTC $\times$ index (Mood et al. 1994) is used (assuming the estimated RTC and calibration index are independent):

$$
\begin{aligned}
\operatorname{var}(\text { RTC } \times \text { index })= & \text { RTC }^{2} \operatorname{var}(\text { index })+\text { index }^{2} \operatorname{var}(\text { RTC }) \\
& \quad+\operatorname{var}(\text { index }) \cdot \operatorname{var}(\text { RTC }) \\
= & 5.71^{2} \times 0.78+1.74^{2} \times 29.23+0.78 \times 29.23 \\
= & 136.19
\end{aligned}
$$

The half width of the $95 \%$ confidence interval of the calibrated RTC is therefore:

$$
\text { CI } \text { half-width }
$$

$$
\begin{aligned}
& =t_{0.05 / 2,9} \times \mathrm{se} \\
& =2.26 \sqrt{(136.19 / 10)} \\
& =8.34
\end{aligned}
$$

The $95 \%$ confidence interval for the calibrated RTC is $1.59 \%$ to $18.28 \%$, more than twice as wide as the original un-calibrated RTC 95\% confidence interval (Fig. 2).

In this example the confidence interval of the calibrated RTC actually included all of the original, un-calibrated RTC confidence interval. Nothing has been gained by using a calibration index and, in fact, what has been lost is precision - the confidence interval is wider. Two approaches can be used to improve this and to make the idea of a calibration index appear more attractive. The first is not recommended. This is to ignore that the calibration index is an estimate and to simply multiply up the RTC from raised-sets by a "fudge factor". The confidence interval of the "fudged" RTC is calculated by using the variance of the original raised-set RTC. We deliberately use the term "fudge-factor" because that is what this approach is. It is misleading and poor practice.

The second approach is to conduct a large study with the aim of quantifying a calibration index. If the study were large enough the variance of the estimated calibration index may be small. With a small variance for the calibration index, the width of the confidence interval of the calibrated RTC would not increase excessively as it did in our example.

We do not recommend this approach either. The costs of such a study would outweigh the advantages of having directly comparable results from ground- and raised-set possum monitoring operations. We recommend that rather than using a calibration index it would be more prudent to specify separate target levels for ground- and raised-set monitoring.

More immediate gains in monitoring consistency can be made by ensuring raised-set 


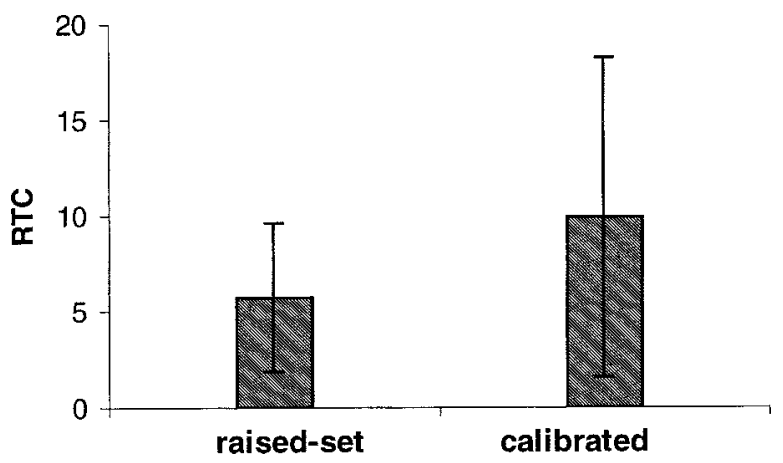

FIGURE 2: Original raised-set and calibrated residual trap-catch (RTC) estimates from the Fiordland 700-mm raised-set data and their associated $95 \%$ confidence intervals.

monitoring operations are comparable. Thomas \& Brown (2000) found that DOC staff used a range of devices and methods to set and lure raised-sets. These need to be standardised.

Finally, in reality, the lack of precision associated with RTC estimates from lowdensity populations make comparisons between areas difficult, regardless of whether they are obtained from raised-sets or not (Brown \& Thomas 2000). The most important issue identified by Brown \& Thomas (2000) for improving possum monitoring was to use larger sample sizes. An enhancement to monitoring is to use alternative, lightweight sampling devices in preference to traps. These, being lighter, mean that many more can be used and this will give larger sample sizes.

\section{ACKNOWLEDGEMENTS}

We would like to thank the Department of Conservation for funding this study. Clare Veltman and Keith Broome (Science and Research Unit, DOC) gave helpful advice and Alan Munn (DOC Fiordland) and Dale Williams (DOC Rotorua) provided assistance for locating study sites. Philip Commins (private research contractor) provided valuable assistance with planning and the implementation of the project in the field, and Christine Bezar gave editorial assistance. Finally we thank the referees for helpful comments on an earlier draft.

\section{REFERENCES}

Brown, J.A.; Hickling, G. 2000: The problems of analysis of pilot-reported bird-strikes as an index for actual bird-strikes at airports. N.Z. J. Zool. 27: 45-47.

Brown, J.A.; Thomas, M.D. 2000: Residual trap-catch methodology for low-density possum populations. University of Canterbury contract report UCDMS 2000/6. 25 p.

Mood, A.M.; Graybill, F.A.; Boes, D.C. 1974: Introduction to the theory of statistics. 3rd edition. McGraw-Hill, New York.

NPCA 2001: Trap-catch for monitoring possum populations. National Possum Control Agencies, Wellington, New Zealand.

Sherley, G.H. 1992: Eradication of brushtail possums (Trichosurus vulpecula) on Kapiti Island, New Zealand: Techniques and methods. Science for Conservation 46. Department of Conservation, Wellington, New Zealand.

Thomas, M.D.; Brown, J.A. 2000: Possum monitoring using raised leg-hold traps. Science for Conservation 164. Department of Conservation, Wellington, New Zealand.

Thomson, C.; Warburton, B.; Moran, L. 1996: Weka- and kiwi-safe trap sets. Landcare Research contract report LC9596/130. 13 p. 